Corrigendum

\title{
Corrigendum to: "Genome-Wide Analysis of SNPs Is Consistent with No Domestic Dog Ancestry in the Endangered Mexican Wolf (Canis lupus baileyi)"
}

Journal of Heredity, advance access publication: 21 March 2018

https://doi.org/10.1093/jhered/esy009

"A new Supplementary Table S1 has been uploaded to replace the previous file. This new file contains corrected museum voucher numbers for samples from the Museum of Southwestern Biology in place of the AZA studbook numbers that were originally included." 